\title{
Mending and malignancy
}

\section{Carcinogenesis and tissue repair: how might chronic tissue injury lead to the development of cancer?}

Philip A. Beachy, Sunil S. Karhadkar and David M. Berman

T he familiar sensation of heartburn whether from greasy food or too much coffee - is caused by acid reflux from the stomach into the oesophagus and is probably harmless on an occasional basis. On the other hand, persistent heartburn can indicate lasting damage to the lining of the oesophagus, resulting in changes in the cellular architecture of the epithelium and an increased incidence of the lethal cancer oesophageal adenocarcinoma. Increased cancer risk is also associated with other types of chronic tissue injury, including those caused by toxins (such as alcohol, cigarette smoke and solvents), chronic infection of Helicobacter pylori and other pathogens, and inflammatory conditions, such as sclerosing cholangitis and inflammatory bowel disease. Genetic mutations are thought to be at the root of cancer, but although some of these injurious agents are mutagens, others are not. How, then, does injury contribute to cancer risk?

To answer this question, we consider tissue response to injury. When faced with injuries that might compromise their function as barriers, the epithelial linings of organs (such as the airway and digestive tract) respond rapidly to restore their integrity. This repair programme includes movement of cells to patch defects, as well as rapid production of cells that can differentiate to form the specialized cells needed to repopulate the injured epithelium. The ultimate source of these differentiated cells within the injured tissue is stem cells.

Stem cells are also increasingly being viewed as playing a role in cancer. Their capacity for self-renewal and unlimited replication make them appealing candidates as the cell of origin for cancer. In addition, stem cells are long-lived, providing ample opportunity to accumulate the mutations that could cause an increase in the rate of cell proliferation and produce clinically significant cancers. There is some evidence to support these ideas. In acute myelogenous leukaemia, the small fraction of leukaemic cells capable of propagating the cancer are undifferentiated, with a combination of surface markers similar to those of blood stem cells found in bone marrow. Elsewhere in the body, the discovery of multipotent progenitor cells that have the capacity for self-renewal opens the possibility that other cancer types also could arise from their respective tissue stem cells.

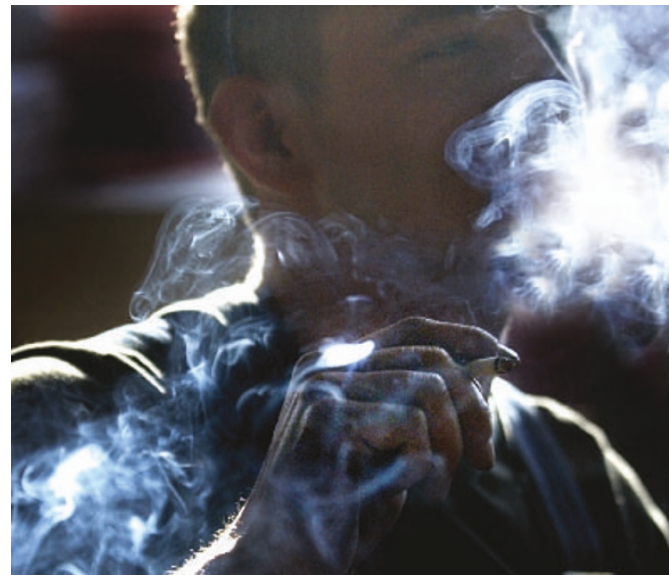

Adding insult to injury: can tissue damage lead to cancer?

Another common trait of both cancer and the activated state of repair is the activation of signalling pathways best known for their roles in embryonic growth and patterning. These include the Hedgehog ( $\mathrm{Hh})$ and Wnt signalling pathways, which also act post-embryonically to stimulate stem cell self-renewal. The Hh and Wnt pathways are also chronically activated in, and required for, the growth of many cancers, including carcinomas that arise in the oesophagus, stomach, lung, pancreas, biliary tract, colon and prostate - which together account for as many as one-third of cancer deaths.

Thus, activation of the Hh and Wnt signalling pathways not only promotes stem cell self-renewal during tissue regeneration, but also the growth of cancers, which may arise from stem cells. Could it be that cancer represents the continuous operation of an unregulated state of tissue repair associated with chronic activation of pathways such as $\mathrm{Hh}$ and Wnt? At the cellular level, the simplest form of this idea would be that a cancer is initiated when a stem cell activated by a regenerative programme fails to return to the resting state once the repair is completed. The events that lock this cell in an activated state and produce a clinically significant cancer could include multiple genetic and/or epigenetic changes, as well as influences from surrounding tissues. The motile behaviour of cells in this activated state, which helps to patch injury-induced epithelial defects, may help to account for the highly invasive nature of some of these epithelial cancers (although it is not yet clear whether epithelial stem cells themselves migrate).

How does this hypothesis link chronic injury to cancer, and how can differences in carcinogenic efficiency of injurious agents be explained? The overall rate of cancer formation would be predicted to depend on the number of activated stem cells that can initiate cancer growth.

Any repeated stimulus leading to a chronic increase in stem-cell activation over time should therefore result in a higher overall frequency of cancer formation. This stimulus could come from exogenous toxins — such as alcohol, which causes liver injury and an increased rate of liver cancer - or from endogenous factors, such as gastric acid, which can cause oesophageal injury and an increased rate of oesophageal cancer. But it could also come from normal physiological events, such as injury to the ovarian epithelium during ovulation, the suppression of which by multiple pregnancies or oral contraceptive use is associated with reduced rates of ovarian cancer. In potent carcinogens such as cigarette smoke, the presence of mutagens in addition to injurious agents could accelerate the oncogenic process by expanding activated stemcell pools while simultaneously inducing genetic changes in these cells. A combination of expanded stem-cell pools and mutagenesis might also account for the efficiency of carcinogenesis in tumour initiator/promoter models, which combine mutagenic agents such as ethylnitrosourea with agents that induce cell proliferation, such as phorbol esters. A better understanding of the response to epithelial injury, in particular the signals that regulate the activation of tissue stem cells, might lead to useful strategies for cancer prevention and therapy.

The authors are affiliated with the Department of Molecular Biology and Genetics and the Howard Hughes Medical Institute (P.A.B. and S.S.K.), and with the Departments of Oncology (P.A.B. and D.M.B.), Pathology (S.S.K. and D.M.B.) and Urology (D.M.B.) at The Johns Hopkins University School of Medicine, Baltimore, Maryland 21205, USA.

\section{FURTHER READING}

Taipale, J. \& Beachy, P. A. Nature 411, 349-354 (2001). Reya, T. et al. Nature 414, 105-111 (2001).

Coussens, L. M. \& Werb, Z. Nature 420, 860-867 (2002).

Al-Hajj et al. Proc. Natl Acad. Sci. USA 100, 3983-3988 (2003).

Watkins, D. N. et al. Nature 422, 313-317 (2003).

Karhadkar, S. S. et al. Nature advanced online publication,12 September 2004

(doi:10.1038/nature02962). 